\title{
Discoidin Domain Receptors Role in Human Diseases
}

\author{
Iker BADIOLA \\ University of the Basque Country, Faculty of Medicine, Department of Cell Biology, Bo Sarriena sn 48940, Leioa Bizkaia, Spain; iker.badiola@ehu.es
}

\begin{abstract}
Discoidin Domain Receptor 1 and Discodin Domain Receptor 2 are the two only members of the DDR family. The DDR family is a Tyrosine Kinase Receptor (TKR) family with some peculiarities compared with other Tyrosine Kinase Receptors such as their natural ligand; which in this case is the fibrillar collagen; or the slow phosphorylation pattern. These peculiarities confer a special role to the receptors present in many diseases development processes as cancer, cirrhosis or lung fibrosis. In this review it is described the overview of the DDRs structure and their role in the different disease development and the possibility to consider them as therapeutic targets.
\end{abstract}

Keywords: cancer, cirrhosis, collagen receptor, metastasis, lung fibrosis, tyrosine kinase receptors

\section{DDR family}

During the study of tyrosine kinase receptors in the tumor cell signalling it was described the presence of a new family of receptors, the DDRs.

The peculiarity of this family is the presence of a homology domain to discoidin I. Discodin I of Dictyostelium discoideum is a lectin implicated in the cellular morphology and cellular aggregation (Devine et al., 1982). Until now it has been described two members of the DDR family: DDR1 and DDR2 (Vogel, 1999). Both have fibrillar collagen type I and III as ligands, but they can also attach to the collagens type II and V (Shrivastava et al., 1998; Vogel et al., 1997). The triple helical configuration of the collagen is essential for receptor activation but the glycosilation of the collagen is only essential in the case of the DDR2 (Vogel et al., 1997). One of the most peculiar characteristics of DDR receptors is the slow phosphorilation that in the other hand persist in the time more than other tyrosine kinase receptors (Vogel et al., 1997).

\section{Discoidin Domain Receptor 1 (DDR1)}

DDR1 is expressed mainly during embrionary development and in the adult tissue in the lung, liver, colon, mammary glands, intestine and brain. Additionally it is very common to find DDR1 in many types of tumors such as: mammary, brain, lung or colon (Laval et al., 1995; Ram et al., 2005; Sanchez et al., 1994; Vogel et al., 2000, 2001; Weiner et al., 2000).

In the DDR1 deficient mice we can see the critical role of the receptor during the postnatal development and in the mammary gland formation (Vogel et al., 2001). It has been described an aberrant formation of the basal renal membrane and a reduction of the cell signalling capacity in the mesenglial cells (Curat and Vogel, 2002). The mus- cular cells derived from DDR1 deficient mice show reduction of migration and chemiotactic capacity compared to with wild type ones. (Hou et al., 2001, 2002)

\section{Discoidin Domain Receptor 2 (DDR2)}

DDR2 is expresed in the muscle, heart, kidney, lung, liver and skin (Alves et al., 1995; Karn et al., 1993; Lai and Lemke, 1994; Olaso et al., 2001, 2002) Recently, it has been described also in the stromal cells surrounding the lung and ovarian tumors (Alves et al., 1995).

In vitro activation of DDR2 induces the expression of MMP1 and MMP (Olaso et al., 2001; Vogel et al., 1997), two enzymes involved in the remodelation of the extracellular matrix and tissue repair (Werb, 1997). The mitogenic response of some growth factors is regulated by metaloproteinases which control the availability of the receptor (Dong et al., 2004). The activation of DDR2 can degrade the extracellular matrix allowing some signalling cascades to interact with other signalling systems (Labrador $e t$ al., 2001). DDR2 phosphorylation occurs during hepatic stellate cell activation and it mediates MMP2 production in response to fibrillar collagen (Olaso et al., 2002). The activation of hepatic stellate cell and the induction of DDR2 by fibrillar collagen produce a feed-back effect resulting in more fibrillar collagen and stronger receptor phosphorylation (Olaso et al., 2002). DDR2 and MMP13 are overexpressed in arthritis, allowing cartilage degradation $(\mathrm{Li} e t$ al., 2005). MMP1 is another metalloproteinase regulated by DDR2 (Vogel et al., 1997). Another important role of DDR2 is the mediation in the migration and proliferation of the hepatic stellate cells and fibroblast via MMP2 regulation (Olaso et al., 2001, 2002).

The DDR2 deficient mice are viable but they are dwarf and their long bones are shorter. This phenotype is due to a lower proliferation of condrocytes during bone growth 
8

(Labrador et al., 2001). Also, skin fibroblast derived from DDR2 deficient mice proliferate at a slower rate than wild type ones, which may explain the observed deficiencies in the wound healing (Olaso et al., 2001, 2002).

\section{Gen structure of DDRs}

The ddr1 gene was cloned for the first time in 1993 through hybridization of a consensus sequence of tyrosine kinase domain of Tirosine Kinase Receptors (Johnson et al., 1993). The homologous gene in mouse called NEP was identified in the same year by Zerlin (1993). At the same time ddr 1 was identified by other groups and was called like trae (Di Marco et al., 1993), PTK-3 (Sanchez et al., 1994), RTK6 (Laval et al., 1995), Cak (Perez et al., 1994) and MCK (Alves et al., 1995).

The human gene ddr1 is localized in the chromosome 6p21.3 near to HLA genes. In the mouse the gene ddr 1 is localized in the chromosome 17. Both mouse and human ddr 1 are composed by 17 exons. The extracellular domain is codified by 8 exons, 3 exons codify for the discoidin domain. An exon codifies for transmembrane domain, 3 for the juxtamenbrane and 5 for the catalytic domain. Compared to other tyrosine kinase receptors, the juxtamenbrane region of DDR1 is longer. 5 isoforms have been described so far generated by alternative splicing of the human ddr 1 gene (Alves et al., 2001). The longer isoform is c with 919 aminoacids, $\mathrm{b}$ is shorter and lacks 6 aminoacids in the tyrosine kinase domain. The isoform $\mathrm{d}$ is the shortest one with only 509 aminoacids and lacks the tyrosine kinase domain. Finally, the isoform e has the tyrosine kinase domain but is not functional (Vogel et al., 2001).

Ddr2 was cloned in 1993 by Karn. At the same time it was cloned by some other groups calling it CCK-2 (Alves et al., 1995), Tyro 10 (Lai and Lemke, 1994), or TKT.

The human gene ddr2 is localized in the chromosome 1 in the region 1q12-q23 and in the mouse in the chromosome 1 in the region $190.0 \mathrm{c} \mathrm{M}$. In humans it is composed by 19 exons and in mouse by 18 .

\section{Protein structure of DDRs}

\section{The DDRs are formed by different regions:}

\section{Extracellular region: discoidin domain and stalk region}

The first 20 aminoacids are the signal peptide in both receptors. Then there is the discoidin domain constituted by 160 aminoacids. In between the discoidin domain and transmembrane domain there is the stalk region (aminoacids 199-412) (Curat et al., 2001). There is no homology of this region with other proteins. The interaction of the discoidin domain with the collagen requires the previous dimerization of the receptor (Letinger, 2003), and this is not the classical concept of TKR activation where the dimerization occurs after the ligand binding to the receptor
(Schlessinger, 2000). The discoidin domain is essential for DDR binding to collagen because in this domain there are some critical residues like Ser-52-Thr57, Arg-105-Lys-112 and Ser-175 (Abdulhussein et al., 2004).

In the stalk region there are 3 important regions: the glicosilation region (Curat et al., 2001) for both receptors, the protease target sequence for DDR1 (Vogel, 2002), and for DDR2 an antigen sequence A5 (Lai and Lemke, 1994). The glicosilation of DDR1 is very important for the signalling and it occurs in the stalk region (Curat $e t$ al., 2001). After the binding to the collagen, DDR1 can be hydrolyzed in two regions: one region beta of $62 \mathrm{kDa}$ attached to the membrane and another region alfa of 54 $\mathrm{KDa}$ which is soluble (Abdulhussein et al., 2004). The biological implication of this feature is still unknown but it may be that the alpha region binds collagens and regulates the union of collagen to the functional receptors.

\section{Juxtamembrane domain}

The juxtamembrane region of DDR 1 and DDR2 is longer compared to the other TKR. There is an identical region for DDR1 and DDR2 in the exon 12 suggesting that it could be a critical region for signal transduction (Alves et al., 2001). In the case of DDR1 the juxtamenbrane region has 176 aminoacids, is rich in prolins and contains 6 tyrosine residues that can be phosphorylation regions, and in the case of DDR2 is similar but shorter (Li et al., 2005). In this region there are some sequences very important for adaptors proteins. This region suffers the different splicing process in the case of DDR1 and is different depending on the isoforms (Alves et al., 1995). The isoform b has an insertion of an exon (exon 11, 111bp) and codified for 37 aminoacids (Playford et al., 1996). This region exists also in the DDR2 and contains the LXNPXY motiv, which is important for Shc binding (Curat et al., 2001; Foehr et al., 2000). DDR1a does not have this region but has a motiv of Shc union (Playford et al., 1996). So DDRla and DDR1b are able to transmit the signal inside the cell recruiting the adaptors proteins (Foehr et al., 2000), but still are unknown the following proteins in the cascade. It has been described in different studies that DDRla and DDR1b have different biological functions (Ardeshna et al., 2000; Bhatt et al., 2000; Hacker et al., 1998; Vogel et al., 2001).

\section{Citosolic region or intracellular: tyrosine kinase domain and $C$-terminus}

The cytoplasmic region contains 438 aminoacids (438911) and includes the kinase domain (Foehr et al., 2000; Playford et al., 1996). This domain is identical in a 39\% to the kinase domains of neurotrophins $(\operatorname{trk} \mathrm{A} / \mathrm{B} / \mathrm{C})$ and shares some characteristics with these receptors (Koo et al., 2006).

The phosphorylation of the DDR receptors is very slow, at least 2 hours; this is one of the most important 
characteristics of DDRs, which is not usual in the receptors tyrosine kinases (Ram et al., 2005; Shrivastava et al., 1998).

The C-terminus region is unusually short for a TKR and contains only 9 aminoacids (Ram et al., 2005).

\section{DDRs signalling}

DDR1 and DDR2 have 13 and 15 tyrosine kinase residues, respectively, in the cytoplasmic region that can be phosphorylated after collagen activation (Foehr et al., 2000; Playford et al., 1996). DDR1 signalling cascade differs with the cell type. In macrophages, phosphorylation occurs via Shc, TRAF6, p38 and NFk- $\beta$ (Matsuyama $e t$ al., 2003). In the mammary epithelial cells, the signalling cascade takes place via Stat 5,while in the tumor mammary cells the signal comes from another receptor: Frizzleed (Dejmek et al., 2005).

Up to date, DDR2 signalling is rather unknown. Recent studies had revealed that DDR2 signalling requires the union of Shc A and Src (Ikeda et al., 2002).

\section{DDRs functions}

The unique ligand described of DDRs is the fibrillar collagen, a key component of the extracellular matrix. Therefore, the cellular functions of DDRs are directly related to the extracellular matrix. In vitro studies of different cell types derived from DDR1 and DDR2 deficient mice show that both receptors are important in the interaction between the cells and the extracellular matrix and are involved in: 1) Cellular adhesion and proliferation (Curat and Vogel 2002; Olaso et al., 2002; Vogel, 2002); 2) cell migration (Hou et al., 2001), and 3) extracellular matrix degradation via MMP activation (Hou et al., 2002; Olaso et al., 2002).

\section{DDRs role in development}

Although DDR1 and DDR2 deficient mice are viable, it is obvious that the expression of these genes is extremely important for the normal development (Labrador et al., 2001; Vogel et al., 2001).

The DDR1 deficiency produces problems in mammary gland formation, kidney and muscular development, directly related with cell proliferation and chemotaxis $(\mathrm{Cu}-$ rat and Vogel 2002; Hou et al., 2002; Vogel et al., 2001). Regarding the DDR2 absence, the knock-out mice are dwarf and it is due to the decrease of chondrocytes proliferation capacity during long bones formation (Labrador et al., 2001).

\section{DDRs in liver cirrhosis}

The fibrotic stage is previous to the liver cirrhotic disease and it is characterized by the extracellular matrix remodel- ling (Friedman, 2003), MMP production (Olaso and Vidal-Vanaclocha, 2003), and fibrillar collagen accumulation (Tsukada et al., 2003). The transdifferentiation of hepatic stellate cells (HSCs) into myofibroblasts is a central event in the fibrogenic responses to hepatic injury induced by non-neoplastic (Friedman, 2003) and neoplastic processes (Amann et al., 2003; Ju et al., 2009; Matsusue et al., 2009). Major features of fibrogenic HSCs are the expression of myofibroblastic marker smooth muscle cell actin (SMA) and tyrosine kinase receptors such as PDGFR- $\beta$ (Wong et al., 1994) and discoidin domain receptor 2 (DDR2) (Olaso et al., 2001); the proliferation and migration into areas of tissue injury (Ikeda et al., 1999), the extracellular matrix production and remodelling (Tsukada et al., 2006), and the secretion of multiple soluble factors that regulate the migration and proliferation of other cell types including liver sinusoidal endothelium cells (LSECs) (Mendoza et al., 1998), parenchymal cell progenitors (Bhatia et al., 1999) and even cancer cells (Desmoulière et al., 2004; Mendoza et al., 1998; Olaso et al., 2003). It has been demonstrated that DDR2 signals for the HSC transdifferntiation process (Olaso et al., 2003). The lacking of DDR2 in the hepatic stellate cells reduce the fibrillar collagen and MMP production (Olaso et al., 2001), therefore the downregulation of DDR2 reduces the fibrotic characteristics of HSC in vitro (Olaso et al., 2002). The critical role of the hepatic stellate cells transdifferentiation during liver fibrosis development and the specific activity of DDR2 in this process make the cell and the receptor good candidates as therapeutics targets for future therapies.

\section{DDRs in lung fibrosis}

Until now only DDR1 has been described in the lung fibrosis (Avivi-Green et al., 2006). The activation of the fibroblast due to external insults is the key event in the lung fibrosis. The presence of the DDR1 in this cells and the surrounding stroma has been described in different studies (Matsuyama et al., 2005, 2006a). In the case of the DDR1 knock-out mice, bleomycin-induced lung fibrosis developed slower than the wild types and it was directly related with less myofibroblast proliferation and migration capacity (Avivi-Green et al., 2006). About the surrounding stroma the DDR1 was localized in the bronchoalveolar lavage fluid and it was reported an important increase of MMP-9, IL-10 and MCP-1 related with an inflammatory microenvironment (Matsuyama et al., 2005). In the study of the DDR1 implication in the lung fibrosis it has been tested a kind of therapeutic approach using small interference RNA. Several siRNA were administrated to bleomycin-induced lung fibrotic mice and the fibrosis decreased in these animals compared with the wild types. The reduction of inflammatory cells, cytokines and collagen deposition were statistically significant (Matsuyama et al., 2006b). 
10

\section{DDRs in cancer disease}

DDR1 has been described usually as a receptor expressed for several tumor cell types such as mammary, brain, lung or colon (Day et al., 2008; Park et al., 2007; Yan et al., 2010). This feature makes sense with the different signalling processes described by the DDR1 in the healthy tissue. The cell proliferation capacity (Olaso et al., 2002, 2003), migration ability (Olaso et al., 2001), and extracellular matrix degradation (Olaso et al., 1997, 2002), are essential characteristics of the cancer cells. The deregulation of this capabilities are tightly related with tumor progression and bad prognosis. For this reason it is reasonable to find an overexpression of DDR1 in several tumor cell types giving to the cancer cells their malignant characteristics.

In the case of DDR2 and cancer, most of the findings have been related with the tumor surrounding stroma (Olaso et al., 2001). The tumor metastasis capacity is not only related with the malignant characteristics of the tumor cells, also the tumor surrounding stroma is important for the metastatis development (Olaso and Vidal-Vanaclocha, 2003). This fact is the so called tumor microenvironment or seed and soil theory (Fidler, 2003). The tumor microenvironment theory says that the tissues hosting the tumor has to be special and specific to support the tumor and during the disease development this tissue suffers changes that produces a special inflammatory status helping the metastatic process (Mendoza et al., 1998). This process has been well described in the liver metastasis and the hepatic stellate cell transdiferentiation in myofribroblast phenotype is the pivotal activity (Friedman, 2003; Olaso et al., 1997). At the same time the role of the DDR2 has been described in this HSC activation process and therefore the relation of DDR2 with the tumor microenvironment and the hosting tissue support to metastatic process is direct (Friedman, 2003).

But recently DDR2 also has been described in melanoma cell lines (Badiola et al., 2011). In vitro experiments described that downregulation of the DDR2 expression in the melanoma cell lines reduces their proliferation and migration capacity and in vivo studies show a considerable reduction of the metastatic capacity when DDR2 expression was partially silenced.

On the other hand it is important to consider the distinctive characteristics of DDRs respect to other TKRs. The low phosphorylation and the feed-back capacities. The phosphrolytaion of the receptors is slow compared with other TKRs but at the meantime the phosporylation produces more fibrillar collagen production which finally is the natural ligand, producing an interesting loop which appears frequently in chronic diseases. This is the case of chirrotic, fibrotic and cancer disease, all of them chronic disease related with slow but constant signalling receptors that perpetuate the processes.

\section{References}

Abdulhussein R, McFadden C, Fuentes-Prior P, Vogel WF (2004). Exploring the collagen-binding site of the DDR1 tyrosine kinase receptor. J Biol Chem 279(30):3146231470 .

Alves F, Vogel W, Mossie K, Millauer B, Hofler H, Ullrich H (1995). Distinct structural characteristicsof discoidin I subfamily receptor tyrosine kinases and complementary expression in human cancer. Oncogene 10(3):609-618.

Alves F, Contag S, Missbach M, Kaspareit J, Nebendahl K, Borchers U, Heidrich B, Streich R, Hiddemann W (2001). An orthotopic model of ductal adenocarcinoma of the pancreas in severe combined immunodeficient mice representing all steps of the metastatic cascade. Pancreas 23(3):227-235

Amann T, Bataille F, Spruss T (2003). Activated hepatic stellate cells promote tumorigenicity of hepatocellular carcinoma. Cancer Sci 100:646-653.

Ardeshna KM, Pizzey AR, Devereux S, Khwaja A (2000). The PI3 kinase, p38 SAP kinase, and NF-kappaB signal transduction pathways are involved in the survival and maturation of lipopolysaccharide-stimulated human monocyte-derived dendritic cells. Blood 96(3):1039-1046

Avivi-Green C, Singal M, Vogel WF (2006). Discoidin domain receptor 1-deficient mice are resistant to beomycin-induced lung fibrosis. Am J Respir Crit Care Med 174(4):420-427.

Badiola I, Villace P, Basaldua I, Olaso E. (2011). Downregulation of discoidin domain receptor 2 in A375 human melanoma cell line reduces its experimental liver metastasis ability. Oncol Rep 26(4)971-978.

Bhatia SN, Balis UJ, Yarmush ML (1999). Effect of cellcell interactions in preservation of cellular phenotype: cocultivation of hepatocytes and nonparenchymal cells. FASEB J 13:1883-1890.

Bhatt RS, Tomoda T, Fang Y, Hatten ME (2000). Discoidin domain receptor 1 functions in axon extension of cerebellar granule neurons. Genes Dev 14(17):2216-2228.

Curat CA, Eck M, Dervillez X, Vogel WF (2001). Mapping of epitopes in discoidin doamain recpeptor 1 critical for collagen binding. The J Biol Chem 276:45942-45958.

Curat CA, Vogel WF (2002). Dicoidin domain receptor 1 controls Growth and adhesion of mesanglial cells. J Am Soc Nephrol 13:2648-2656.

Day E, Water B, Spiegel K (2008). Inhibition of collageninduced discoidin domain receptor 1 and 2 activation by imatinib, nilotinib and dasatinib. Europ J Pharmacol 599(13):44-53.

Dejmek J, Leandersson K, Manjer J, Bjartell A, Emdin SO, Vogel WF, Landberg G, Andersson T (2005). Expression and signaling activity of Wnt-5a/discoidin domain receptor-1 and Syk plays distinct but decisive roles in breast cancer patient survival. Clin Cancer Res 11(2 Pt 1):520-528. 
Desmoulière A, Guyot C, Gabbiani G (2004). The stroma reaction myofibroblast: a key player in the control of tumor cell behavior. Int J Dev Biol 48:509-517.

Devine JM, Tsang AS, Williams JG (1982). Differential expression of the members of the discoidin I multigene family during growth and development of Dictyostelium discoideum. Cell 28(4):793-800.

Di Marco V, Lo Iacono O, Capra M, Grutta S, Ciaccio C, Gerardi C, Maggio A, Renda D, Almasio P, Pisa R (1993). Alpha interferon treatment of chronic hepatitis $\mathrm{C}$ in betathalassaemia. Gut 34(2 Suppl):S142-143.

Dong Z, Ghabrial M, Katar M, Fridman R, Berks RS (2004). Membrane-type matrix metalloproteinases in mice intracorneally infected with Pseudomonas aeruginosa. Invest Ophthalmol Vis Sci 41(13):4189-4194.

Foehr ED, Tatavos A, Tanabe E, Raffioni S, Goetz S, Dimarco E, De Luca M, Bradshaw RA (2000). Discoidin domain receptor 1 (DDR1) signaling in PC12 cells: activation of juxtamembrane domains in PDGFR/DDR/TrkA chimeric receptors. FASEB J 14(7):973-981.

Fidler IJ (2003). The pathogenesis of cancer metastasis: The "seed and soil" hypothesis revised. Nat Rev Cancer 3(6):453-458.

Friedman SL (2003). Mechanisms of disease: Mechanisms of hepatic fibrosis and therapeutic implications. Nat Clin Pract Gastroenterol Hepatol 1(2):98-105.

Hacker H, Mischak H, Miethke T, Liptay S, Schmid R, Wagner $\mathrm{H}$ (1998). CpG-DNA-specific activation of antigen presenting cells requires stress kinase activity and is preceded by non-specific endocytosis and endosomal maturation. Embo 17:6230-6240.

Hou G, Vogel W, Bendeck MP (2001). The discoidin domain receptor tyrosine kinase DDR1 in arterial wound repair. The J Clin Investig 107(6):727-735.

Hou G, Vogel WF, Bendeck MP(2002). Tyrosine kinase activity of discoidin domain receptor 1 is necessary for smooth muscle cell migration and matrix metalloproteinase expression. Circ Res 90(11):1147-1149.

Ikeda K, Wakahara T, Wang YQ (1999). In vitro migratory potential of rat quiescent hepatic stellate cells and its augmentation by cell activation. Hepatol 29:1760-1767.

Ikeda K, Wang LH, Torres R, Zhao H, Olaso E, Eng FJ, Labrador P, Klein R, Lovett D, Yancopoulos GD, Friedman SL, Lin HC (2002). Discoidin domain receptor 2 interacts with Src and Shc following its activation by type I collagen. J Biol Chem 277(21):19206-19212.

Johnson JD, Edman JC, Rutter WG (1993). A receptor tyrosine kinase found in breast carcinoma cells has an extracellular discoidin I-like domain. Proc Natl Acad Sci USA 90(12):5677-5681

Ju MJ, Qiu SJ, Fan J (2009). Peritumoral activated hepatic stellate cells predict poor clinical outcome in hepatocellular carcinoma after curative resection. Am J Clin Pathol $131: 498-510$.
Karn T, Holtrich U, Brauningher A, Bohme B, Strebhartdt K (1993). Structure expression and chromosomal mapping of TKT from man and mouse: a new subclass of receptor tyrosine kinases with a factor VIII-like domain. Oncogene 8:3433-3440.

Koo BC, McPoland P, Wagoner JP, Kane OJ, Lohmann V, Polyak SJ (2006). Relationships between hepatitis C virus replication and CXCL-8 production in vitro. J Virol 80(16):7885-7893.

Labrador JP, Azcoitia V, Tuckerman J, Lin C, Olaso E, Mañes S, Bruckner K, Goergen J, Lemke G, Yancopoulus G, Angel P, Klein R (2001).The collagen receptor DDR2 regulates proliferation and its elimination leads to dwarfism. EMBO Rep 2(5):446-452.

Lai C, Lemke G (1994). Structure and expression of the Tyro 10 receptor tyrosine kinase. Oncogene 9(3):877-883.

Laval S, Butler R, Shelling AN, Hanby AM, Poulsom R, Ganesan TS (1995). Isolation and characterization of an epithelialspecific receptor tyrosine kinase from an ovarian cancer cell line. Cell Growth Differ 5:1173-1183.

Letinger B (2003). Molecular Analysis of collagen binding by the human discoidin domain receptors, DDR1 and DDR2: Identification of collagen binding sites in DDR2. J Biol Chem 278(19):16761-16769.

Li W, Zhang YQ, Liu XP, Yao LB, Sun L (2005). Regular expression of discoidin domain receptor 2 in the improved adjuvant-induced animal model for rheumatoid arthritis. Chin Med Sci J 20(2):133-137.

Mao TK, Kimura Y, Kenny PT, Branch A, Gish GR, Kung H, Friedman SL, Gershwin ME (2002). Elevated expression of tyrosine kinase DDR2 in primary biliary cirrhosis. Autoimmunity 35(8):521-529.

Matsusue R, Kubo H, Hisamori S (2009). Hepatic stellate cells promote liver metastasis of colon cancer cells by the action of SDF-1/CXCR4 Axis. Ann Surg Oncol 16:2645-2653.

Matsuyama W, Kamohara H, Galligan C, Faure M, Yoshimura T (2003). Interaction of discoidin domain receptor 1 isoform b (DDR1b) with collagen activates p38 mitogen-activated protein kinase and promotes differentiation of macrophages. The FASEB Journal 23:123-134.

Matsuyama W, Watanabe M, Shrirahama Y, Oonakhonak H, Mitsuyama H. Higashimoto I, Yoshima T, Osama H, Arimura K (2005). Activation of discoidin domain receptor 1 on CD14- positive bronchoalveolar lavage fluid cells induces chemokine production in idiopathic pulmonary fibrosis. J Inmunol 174(10):6490-6498.

Matsuyama W, Watanabe M, Shrirahama Y, Mitsuyama H Higashimoto I, Osama H, Arimura K (2006a). Discoidin Domain Receptor 1 contributes to the survival of lung fibroblast in idiopathic pulmonary fibrosis. Am J Pathol 168(3):866-877.

Matsuyama W, Watanabe M, Shrirahama Y, Hirano R, Mitsuyama H, Higashimoto I, Osama H, Arimura K (2006b). Supresion of discoidin domain receptor 1 by 
12 RNA interference attenuates lung infalmation. J Inmulog 176(3):1928-1936.

Mendoza L, Olaso E, Anasagasti MJ (1998). Mannose receptormediated endothelial cell activation contributes to B16 melanoma cell adhesion and metastasis in liver.J Cell Physiol 174:322-330.

Olaso E, Santisteban A, Bidaurrazaga J (1997). Tumor dependent activation of rodent hepatic stellate cells during experimental melanoma metastasis. Hepatol 26(3):634-644.

Olaso E, Ikeda K, Eng FJ, Xu L, Wang L, Lin H, Friedman SL (2001). DDR2 receptor promotes MMP2-mediated proliferation and invasion by hepatic stellate cells. The J Clin Investig 108(9):1369-1378.

Olaso E, Labrador JP, Wang LH, Ikeda K, Eng FJ, Klein R, Lovett DH, Lin H, Friedman SL (2002). Discoidin domain receptor 2 regulates fibroblast proliferation and migration through the extracellular matrix in association with transcriptional activation of matrix metalloproteinase-2. J Biol Chem 277(5):3606-3613.

Olaso E, Salado C, Egilegor E (2003). Proangiogenic role of tumor-activated hepatic stellate cells in experimental melanoma metastasis. Hepatol 37:674-685.

Olaso E, Vidal-Vanaclocha F (2003). Use of tumor-activated hepatic stellate cell as a target for the preclinical testing of anti-angiogenic drugs against hepatic tumor development. Met Mol Med 85:79-86.

Park HS, Kim KR, Lee HJ (2007). Overexpression of discoidin domain receptor 1 increases the migration and invasion of hepatocellular carcinoma cells in association with matrix metalloproteinase. Oncol Rep 18:1435-1441.

Perez JL, Shen X, Finkernagel S, Sciorra L, Jenkins NA, Gilbert DJ, Wong W (1994). Identification and chromosomal mapping of a receptor tyrosine kinase with a putative phospholipids binding sequence in its ectodomain. Oncogene 9:211-219.

Playford MP, Butler RJ, Wang XC, Katso RM, Cooke IE, Ganesan TS (1996). The genomic structure of discoidin receptor tyrosine kinase. Genome Res 6(7):620-627.

Ram R, Lorente G, Nikolich K, Urfer R, Foher E, Nagavarapu U (2005). Discoidin domain receptor la (DDR1a) promotes glioma cell invasion and adhesion in association with Matrix Metalloproteinase-2. J Neurooncol 23(3):15-23.

Sanchez MP, Tapley P, Saini SS, He B, Pulido D, Barbacid M (1994). Multiple tyrosine kinases in rat hippocampal neurons: isolation of Ptk-3, a receptor expressed in proliferative zones of developing brain. Proc Nat Acad Sci 91:1819-1823.

Schlessinger (2000). Cell signaling by receptor tyrosine kinases. Cell 103(2):211-225.

Shrivastava A, Radziejewski C, Campbell E (1998). An orphan receptor tyrosine kinase family whose members serve as nonintegrin collagen receptors. Mol Cell 1(1):25-34.

Tsukada S, Parsons CJ, Rippe RA (2006). Mechanisms of liver fibrosis. Clin Chim Acta 364:33-60.

Vogel W, Gish GD, Alves F, Pawson T (1997). The discoidin domain receptor tyrosine kinases are activated by collagen. Mol Cell 1(1):13-23.

Vogel WF (1999). Discodin domain receptors: structural relations and functional implications. The FASEB J13(Suppl):S77-82.

Vogel W, Brakebusch C, Fässler R, Alves F, Ruggiero F, Pawson $T$ (2000). Discoidin domain receptor 1 is activated independently of beta (1) integrin.J Biol Chem 275(8):57795784.

Vogel WF, Aszodi A, Alves F, Pawson T (2001). Discoidin domain receptor 1 tyrosine kinase has an essential role in mammary gland development. Mol Cell Biol 21(8):29062917.

Vogel W (2002). Ligand-induced shedding of discoidin domain receptor 1. FEBS Letters 514:175-180.

Weiner HL, Huang H, Zazag D, Boyce H, Litchtembaun R, Ziff EB (2000). Consistent and selective expression of the discoidin domain receptor- 1 tyrosine kinase in human brain tumors. Neurosurgery 47(6):1400-1409.

Werb Z (1997). ECM and cell surface proteolysis: regulating cellular ecology. Cell 91(4):439-442.

Wong L, Yamasaki G, Johnson RJ (1994). Induction of betaplatelet-derived growth factor receptor in rat hepatic lipocytes during cellular activation in vivo and in culture. J Clin Invest 94:1563-1569.

Yan SH, Baek HA, Lee HJ (2010). Discoidin domain receptor 1 is associated with poor prognosis of non-small cell lung carcinomas. Oncol Rep 24(2):311-319.

Zerlin M, Julius MA, Goldfarb M (1993). NEP: a novel receptorlike tyrosine kinase expressed in proliferative neuroepithelia. Oncogene 8:2731-2739. 